\title{
Machine Vision-based Measurement System for Vehicle Body Inspection
}

\author{
András Rövid \\ Óbuda University, John von Neumann Faculty of Informatics \\ Bécsi út 96/B, 1034 Budapest, Hungary \\ rovid.andras@nik.uni-obuda.hu
}

\begin{abstract}
The main contribution of the paper is to propose a system for fast and easy measurement of car body parts to support their inspection. The proposed system is composed from a camera-projector $(\mathrm{CP})$ subsystem and a multi-camera and active marker based tracking system (TS). During the reconstruction process ,"rotating” gray code pattern is projected onto the targeted surface which improves the robustness and accuracy of the measurement. Afterwards based on triangulation the $3 D$ coordinates of surface points are estimated.
\end{abstract}

Keywords: 3D measurement; car-body; projector; point cloud; inspection

\section{Introduction}

Recently the number of applications where the 3D measurement plays crucial role is increasing more and more. As few examples the industrial applications can be emphasized such as product inspection, design and manufacturing of various components, etc. The required accuracy of the measurement and other prescribed properties depend on the particular application. As the technology evolves new possibilities are at hand, new technologies can be applied to improve the performance of the measurement.

There are numerous well known techniques to perform 3D measurement and reconstruct the surface of various objects. Among the most popular and most reliable approaches the structured light and laser based solutions can be emphasized, where a coded pattern, fringe pattern or laser stripe is projected onto the targeted surface and based on pattern analysis and subsequent triangulation the $3 \mathrm{D}$ surface can be reconstructed.

The main difference in these methods lies first of all in the type of projected sequence to solve the correspondence matching problem which is the most crucial issue during the reconstruction. Some techniques are using colors for coding [7], 
while the others are based on binary code, Gray code [9] [10] or fringe pattern projection [1-3] [11-13], etc. Although the projection of pattern sequences is relatively time consuming process, by using high speed cameras and precise synchronization real-time reconstruction can also be achieved. For example certain phase shift based methods - which belong to the family of fringe analysis based solutions - can offer real-time reconstruction [14].

Among the hardware based solutions first of all the utilization of Time of Flight (TOF) cameras may be emphasized [4]. Furthermore one can find applications where the stereo imaging without pattern projection is applied, e.g. robotics, obstacle detection, etc. in which case the point correspondence matching may become ambiguous or imprecise, therefore the achievable precision of the measurement is relatively low. Beside the above mentioned techniques one can find in the literature other interesting approaches, as well. The selection of the most appropriate measurement method depends first of all on the application requirements. The main aim of our investigation was to design and implement a robust, low cost high precision 3D measurement system to ensure the measurement of car body elements and generate data appropriate for their inspection or to perform certain analysis.

Another consideration - related to $3 \mathrm{D}$ reconstruction - was the extension of the measurement range by maintaining the accuracy at acceptable level. There are various methods to achieve this goal, which aim is to extend the „working space” trough tracking the scanning device [8]. This goal can be achieved for example by attaching the scanning device to robot arm or tracking it by external cameras.

The primary application of the proposed system is to support the evaluation process of crash tested vehicles. Crash tested cars provide a lot of important data, which can be obtained through detailed measurements of the car-body. By using these data the mathematical models of deformation processes can be tuned or identified. Since the measurement of critical points manually is a time consuming process the necessity of the $3 \mathrm{D}$ reconstruction of crucial parts is important.

The main aim of the paper is to introduce a method based on machine vision and pattern projection together with a tracking system to enable quick and robust measurement of car body elements.

The paper is organized as follows: Section II describes the architecture of the proposed system, Section III deals with calibration related issues, Section IV describes how the markers used for tracking are detected and identified. Section V describes the reconstruction itself and finally results and conclusions as well as future works are reported. 


\section{The Architecture of the System}

An important consideration during the development of the system was to ensure the acquisition of large objects (car body parts) and hidden or covered surfaces with high precision. For this purpose an active optical scanning technology was combined with a multi-camera based tracking system depicted by Fig. 1. The active optical scanner is composed from a camera and projector which is tracked by the multi-camera system (see Fig. 1). Thus the CP system can be repositioned to ensure the acquisition of complex surfaces, as well. To ensure easy tracking, active LED markers - controlled by the software - have been attached to the CP system. This solution supports their unambiguous identification even if the lighting conditions are not appropriate. The working principle of the system can be decomposed into following steps:

First of all the system has to be calibrated, i.e. the intrinsic and extrinsic parameters of the cameras and that of the projector (considered as an inverse camera model) are determined. At this stage the hand-eye problem has to be solved, as well. After the system calibration process the measurement can be started during which the below steps are performed:

The active markers are detected and identified followed by the estimation of their spatial location used to estimate the relative rotation and transition of the $\mathrm{CP}$ system with respect to an initial reference value.

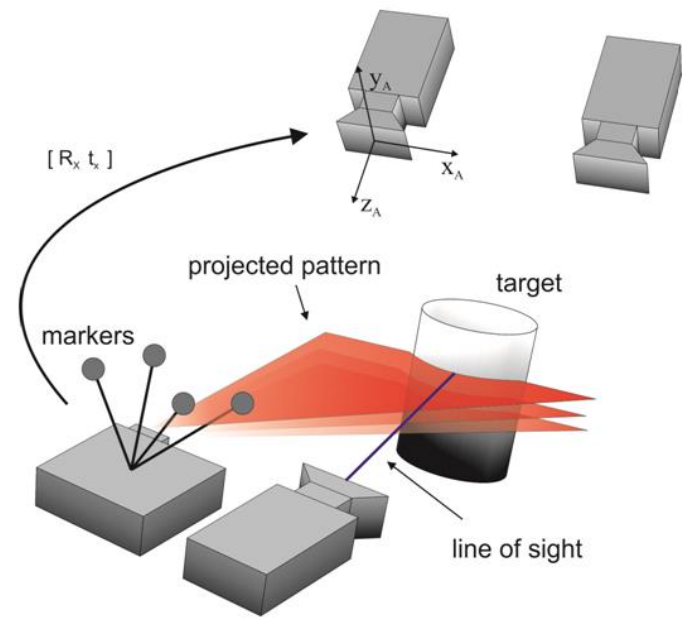

Figure 1

The architecture of the proposed system

Next, the so called rotated Gray code pattern is projected onto the targeted surface and based on ray-plane intersection the 3D coordinates of each projected pixel are determined. Accordingly the obtained 3D points are transformed into the world coordinate system. 
Finally the point cloud is stored in a KD-tree structure and processed, i.e. the noise is eliminated and polynomial fitting is performed. In the upcoming section the main components of the system are described in more detail.

\section{System Calibration}

The calibration plays the most crucial part of the system. Since the proposed system has numerous components (cameras for tracking, projector, cameras for acquiring the illuminated surface), the accuracy strongly depends on the precision of the calibration of the mentioned elements as well as on the accuracy of the estimated relationships of coordinate systems. All these factors have significant impact on the output of the whole system therefore it is important consider them with high priority. The calibration of the system can be decomposed into three main parts, i.e. calibration of cameras; calibration of the projector and estimation of the relation between the probe and the world coordinate system (see Fig. 2).

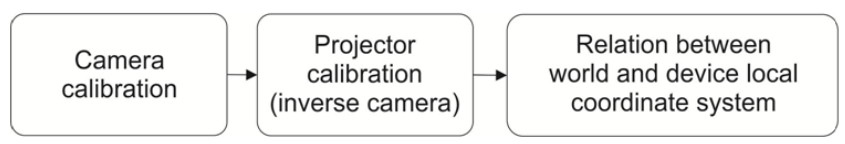

Figure 2

Flow of calibration

\subsection{Camera Calibration}

The intrinsic and extrinsic parameters of the cameras as well as the distortion coefficients of the applied lens were estimated by Zhang's method [5]. As calibration object a chessboard plate was used, which was acquired from various viewpoints by the cameras and based on the $3 \mathrm{D}$ coordinates of its corners and their projections onto the camera image plane the intrinsic as well as the extrinsic parameters of cameras can be determined (for more details see [5]).

\subsection{Projector Calibration}

The calibration of the projector is relatively more complex because in this case the 3D coordinates of the projected chessboard corners have to be estimated separately. The model of projector can be considered as an inverse camera model. As calibration pattern chessboard has been used. In order to estimate the intrinsic and extrinsic parameters of the projector, the first task is to get 3D-2D correspondences. In case of cameras the spatial coordinates of the chessboard 
(calibration object of known dimensions) corners are known, their projections can be seen in the camera image. In case of a projector the situation is opposite, i.e. the coordinates of the corners projected onto a planar surface have to be estimated while the $2 \mathrm{D}$ coordinates of the corners in the image plane of the projector are known in advance.

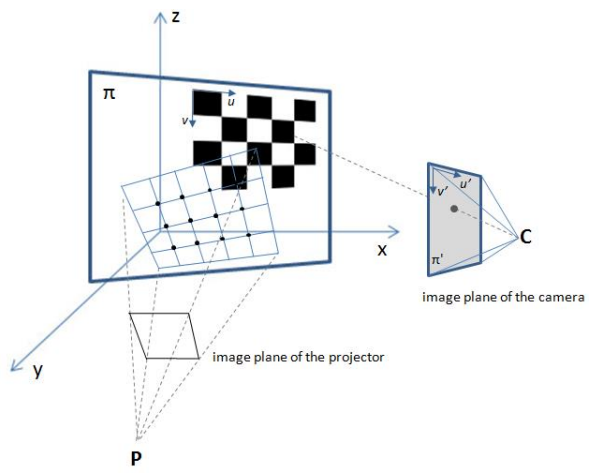

Figure 3

Illustration of projector calibration. ( $\mathbf{P}$ - projector centre; $\mathbf{C}$ - camera centre). First the homography between $\boldsymbol{\pi}$ and $\boldsymbol{\pi}$ ' is estimated. Afterwards the $u, v$ coordinates of projected corners are determined.

For estimating the coordinates of the projected corners first a chessboard pattern with known dimensions is attached to the planar surface. The top most horizontal and left most vertical edges represent the $u$ and $v$ axes of the reference coordinate system (see Fig. 3). As the coordinates of the physical chessboard corners in this coordinate system as well as the coordinates of their projections onto the camera image plane are known a homography can be estimated between the two projective planes [19].

By using the estimated homography the coordinates of the corners projected by the projector onto the planar surface (to which the chessboard pattern is attached) can be determined.

$\left[\begin{array}{c}w u_{b} \\ w v_{b} \\ w\end{array}\right]=\left[\begin{array}{lll}h_{11} & h_{12} & h_{13} \\ h_{21} & h_{22} & h_{23} \\ h_{31} & h_{32} & h_{33}\end{array}\right]\left[\begin{array}{c}u_{a} \\ v_{a} \\ 1\end{array}\right]$,

where $u_{\mathrm{a}}, v_{\mathrm{a}}$ and $u_{\mathrm{b}}, v_{\mathrm{b}}$ stand for corresponding points in planes $\boldsymbol{\pi}$ and $\boldsymbol{\pi}$ ' respectively (see Fig. 3). H stands for the estimated homography matrix.

Accordingly the intrinsic and extrinsic parameters of the projector can be estimated - as in case of cameras - from known $3 \mathrm{D} \rightarrow 2 \mathrm{D}$ correspondences. 


\subsection{Hand-eye Calibration}

The relation between the local coordinate system of the projector-camera subsystem and that of the world can be expressed as follows (see Fig. 4):

$$
\begin{aligned}
& \mathbf{R}_{A} \mathbf{R}_{X}=\mathbf{R}_{X} \mathbf{R}_{B} \\
& \left(\mathbf{R}_{A}-\mathbf{I}\right) \mathbf{t}_{X}=\mathbf{R}_{X} \mathbf{t}_{B}-\mathbf{t}_{A}
\end{aligned}
$$

Here matrices $\mathbf{R}_{\mathrm{A}}, \mathbf{R}_{\mathrm{B}}$ and vectors $\mathbf{t}_{\mathrm{A}}, \mathbf{t}_{\mathrm{B}}$ stand for the transformation between two arbitrary positions and orientations of the projector-camera subsystem in the world coordinate system and the corresponding rotation and transition of the local coordinate system of the device (i.e. the coordinate system of the camera in the projector-camera subsystem).

$\mathbf{R}_{\mathrm{X}}$ and $\mathbf{t}_{\mathrm{X}}$ represent the unknown transformation from the local into the world coordinate system. After few steps of derivation we get the following minimization problem [6]:

$$
\begin{aligned}
& f_{1}\left(\mathbf{R}_{X}\right)=\min _{\mathbf{R}_{X}} \sum_{i=1}^{n}\left\|\mathbf{n}_{A i}-\mathbf{R}_{X} \mathbf{n}_{B i}\right\|^{2} \\
& f_{2}\left(\mathbf{t}_{X}\right)=\min _{\mathbf{t}_{X}} \sum_{i=1}^{n}\left\|\mathbf{R}_{X} \mathbf{t}_{B}-\left(\mathbf{R}_{A}-\mathbf{I}\right) \mathbf{t}_{X}-\mathbf{t}_{A}\right\|^{2}
\end{aligned}
$$

where $\mathbf{n}_{\mathrm{Ai}}$ and $\mathbf{n}_{\mathrm{Bi}}$ stand for the eigenvectors of $\mathbf{R}_{\mathrm{Ai}}$ and $\mathbf{R}_{\mathrm{Bi}}$ corresponding to the unit eigenvalue. $f_{1}\left(\mathbf{R}_{X}\right)$ can be solved for example by using quaternions together with langrange multipliers [6], while $f_{2}\left(\mathbf{t}_{X}\right)$ by least squares method.

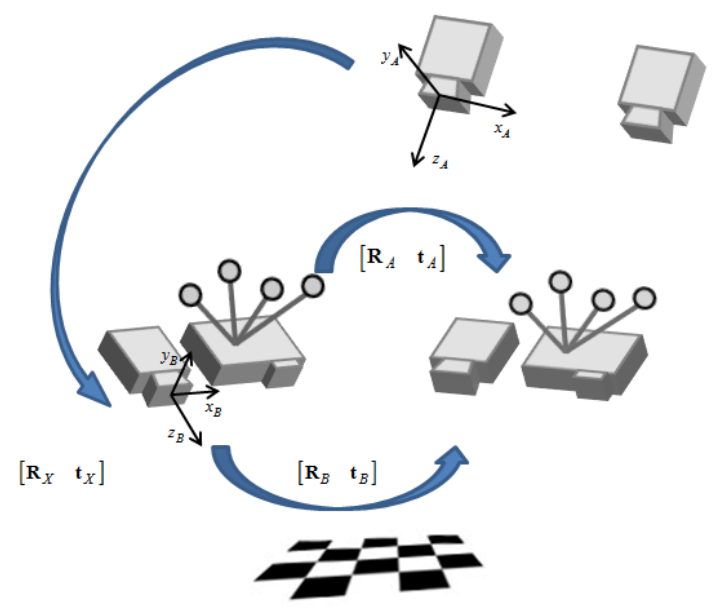

Figure 4

Illustration of the hand-eye problem 


\section{The Features of the Tracking Subsystem}

In order to extend the range of measurement to ensure the reconstruction of large surfaces the camera-projector subsystem has to be tracked and the point clouds obtained from different locations of the CP subsystem can be combined in order to get a complete model and ensure the acquisition of hidden areas, as well. There are various techniques to perform the tracking. The proposed system uses multicamera system to achieve this goal.

The CP subsystem is equipped with sequence coded active LED markers, which are controlled by the software and synchronized with the tracking subsystem. For this purpose a controller has been designed by the authors. The LED markers are turned on and off based on their assigned ID. Each state of the LED in the sequence represents one bit in the ID. An example of marker ID estimation can be followed in Fig. 5.

Let $\mathbf{a}_{\mathrm{i}}$ and $\mathbf{b}_{\mathrm{i}}, i=1 . . N$ represent the $3 \mathrm{D}$ coordinates of markers corresponding to two different locations of the camera-projector subsystem. The transformation between the two states can be obtained as follows:

$f(\mathbf{R})=\min _{\mathbf{R}} \sum_{i=1}^{N}\left\|\left(\mathbf{b}_{i}-\mathbf{q}\right)-\mathbf{R}\left(\mathbf{a}_{i}-\mathbf{p}\right)\right\|^{2}$ and the transition can be obtained simply as: $\mathbf{t}=\mathbf{q}-\mathbf{p}$, where $\mathbf{p}=\frac{1}{N} \sum_{n=1}^{N} \mathbf{a}_{i}$ and $\mathbf{q}=\frac{1}{N} \sum_{n=1}^{N} \mathbf{b}_{i}$.

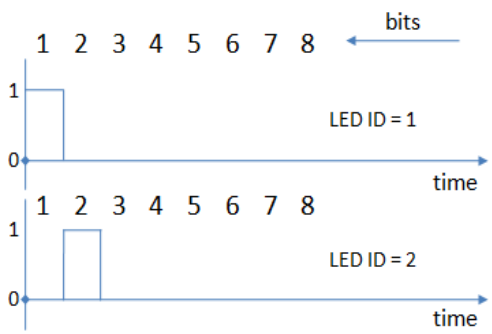

$\begin{array}{llllllll}1 & 2 & 3 & 4 & 5 & 6 & 7 & 8\end{array}$

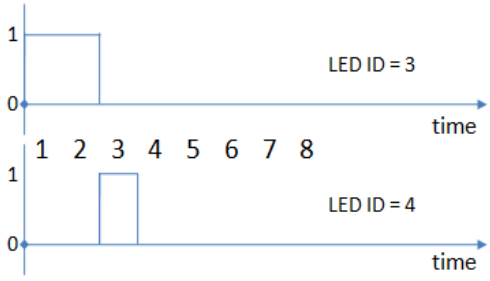

Figure 5

An example how the ID of each LED marker is determined $($ LED ON $=1 ;$ LED OFF $=0$ ) 
The algorithm used to detect the center of gravity of markers [15]:

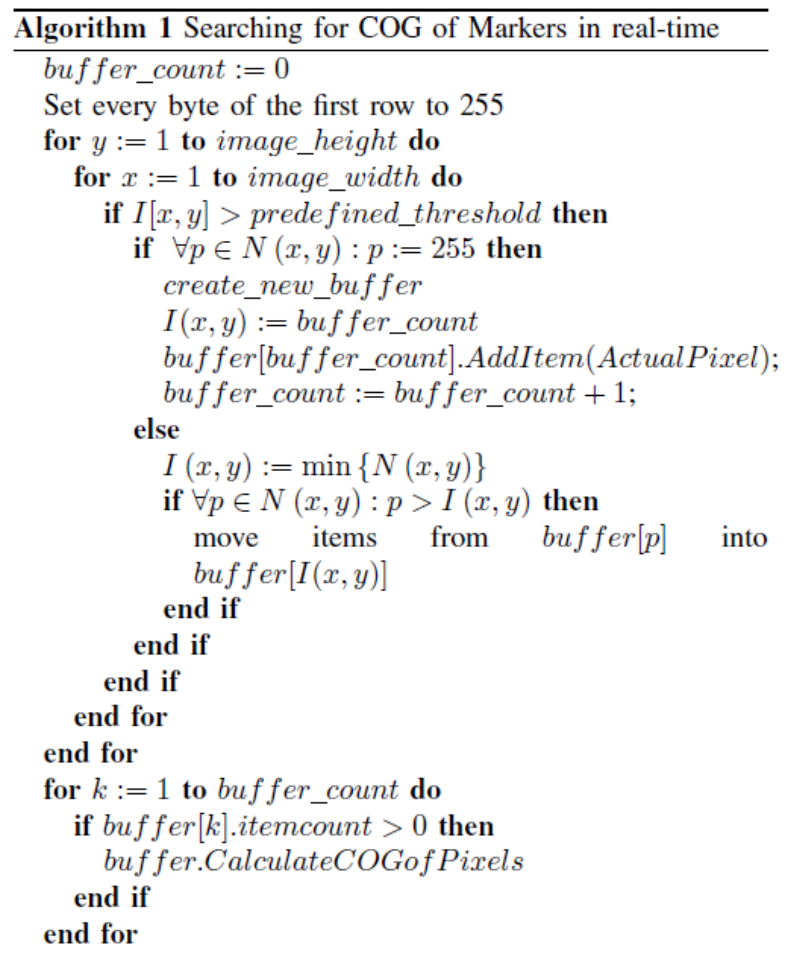

\section{Reconstruction}

The main aim is to determine which projected column the camera sees in a given pixel. To identify the columns and rows in the image plane of the projector Gray coded sequence was projected onto the targeted surface (see Fig. 6). It can easily be recognized that in gray code sequence the consecutive codes differ only in one bit. In noisy images this kind of coding ensures more reliable identification.

The patterns are sequentially projected onto the targeted surface. Such a way for each camera image pixel the corresponding column in the projector image plane can be identified. Afterwards the $3 \mathrm{D}$ coordinates can be determined by the intersection of the line of sight corresponding to the pixel and the plane formed by the projected column (row) affecting the pixel (see Fig. 1).

In order to identify the planes the black and white regions of the projected patterns should be extracted from the camera image. This step is performed by subtracting the original image from the image taken when the target was fully illuminated and subsequently applying binarization based on thresholding. In order to increase the 
robustness against the influence of external light sources, the pattern sequence is projected in various orientations onto the targeted surface. This first of all ensures more reliable acquisition and higher accuracy (see Fig. 7).

The results obtained by using different orientations of the pattern are averaged. The below diagram illustrates the steps of the reconstruction. The gray box represents the task performed by the camera-projector subsystem. The resulted point cloud is stored in KD-tree structure, which is a binary tree useful to organize finite set of points in the $k$-dimensional space [16] [18].

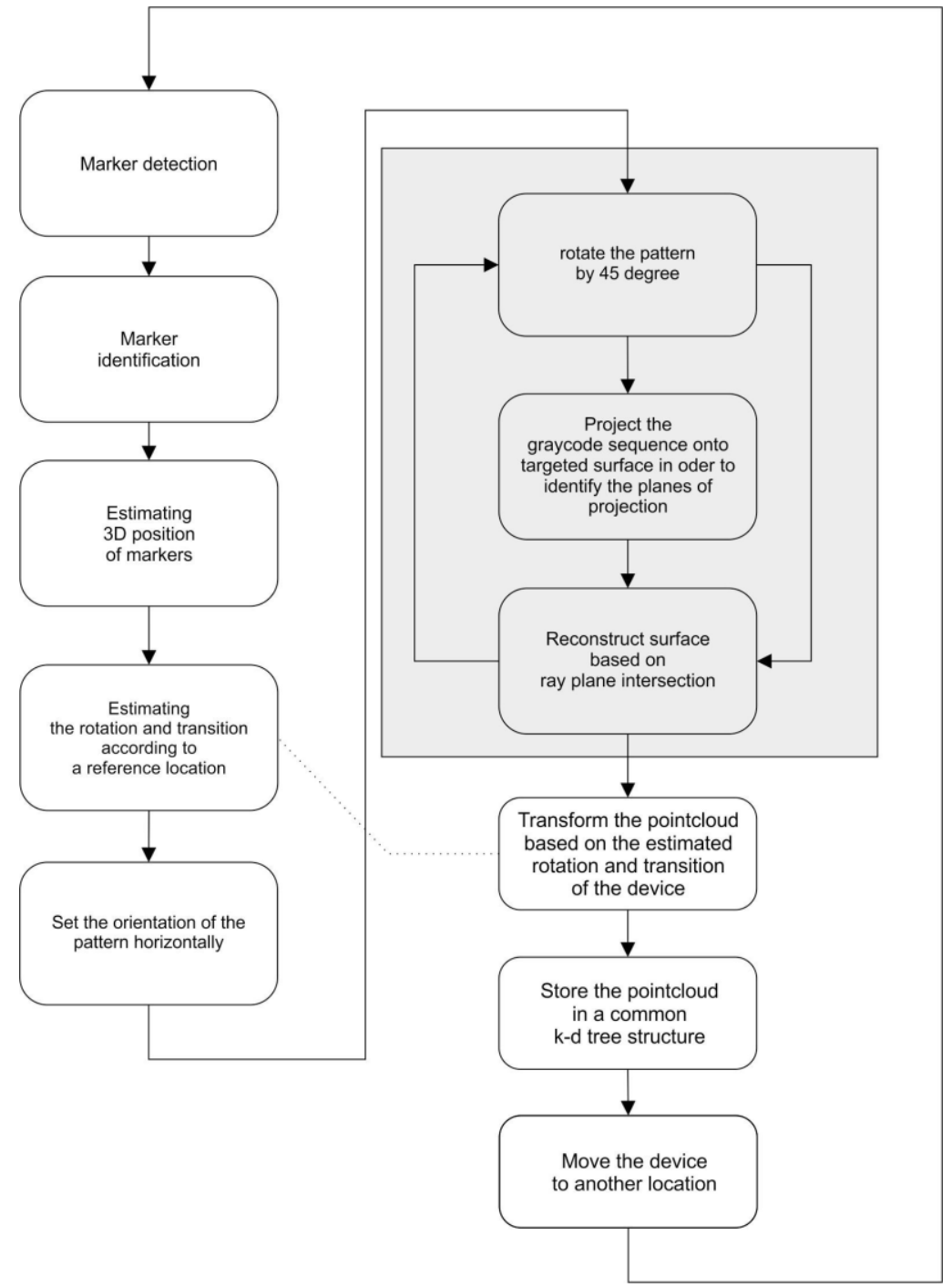




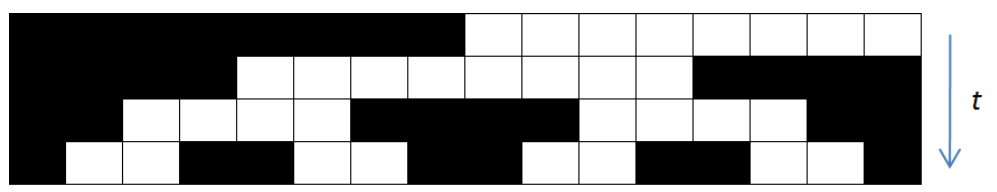

Figure 6

Illustration of a 4 bit gray code sequence. The horizontal patterns are projected onto the targeted surface sequentially (consecutive codes differ in one bit only). In the below example a 10 bit Gray code sequence was used.

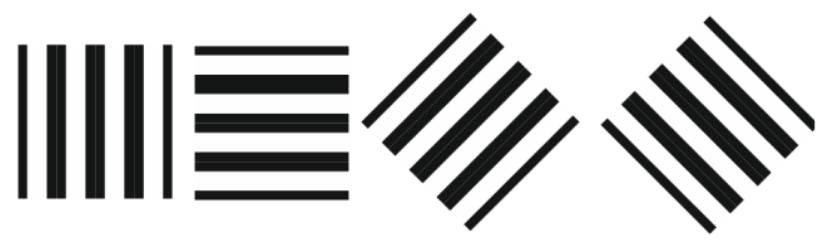

Figure 7

Illustration of the projected pattern in different orientations

After relocating the projector-camera subsystem and performing another measurement from the new viewpoint we obtain a new surface segment which is placed in the same k-d tree structure as the segment from the previous measurement. This procedure has to be repeated in order to measure larger surfaces.

\section{Results}

The result below stands for a point cloud of a car door segment obtained by the proposed system. In Table 1 the specifications of the used hardware can be followed. For visualizing and processing the obtained point cloud data the Point Cloud Library (PCL) has been utilized.

Table 1

\begin{tabular}{|l|l|}
\hline Properties & Value \\
\hline Stereo-camera resolution & $1024 \times 768$ pixels \\
\hline Probe-camera resolution & $1024 \times 768$ pixels \\
\hline Projector resolution & $1024 \times 768$ pixels \\
\hline Frame rate & $30 \mathrm{FPS}$ \\
\hline Number of LED markers & 5 \\
\hline
\end{tabular}




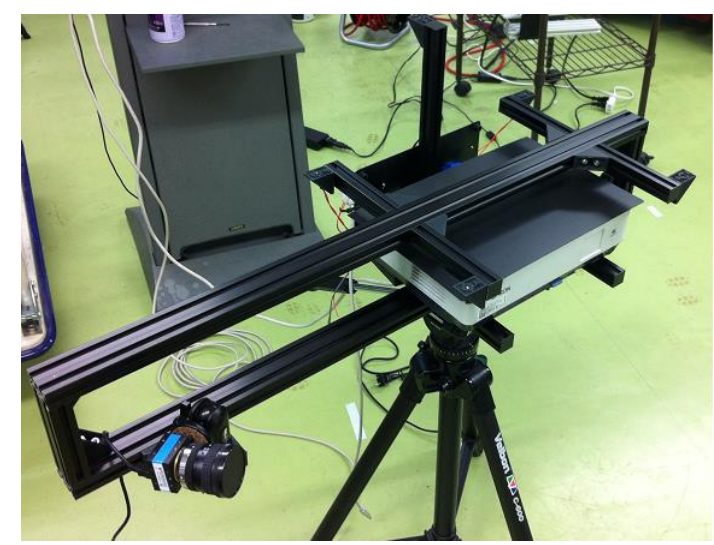

Figure 8

The hardware of the proposed camera-projector subsystem

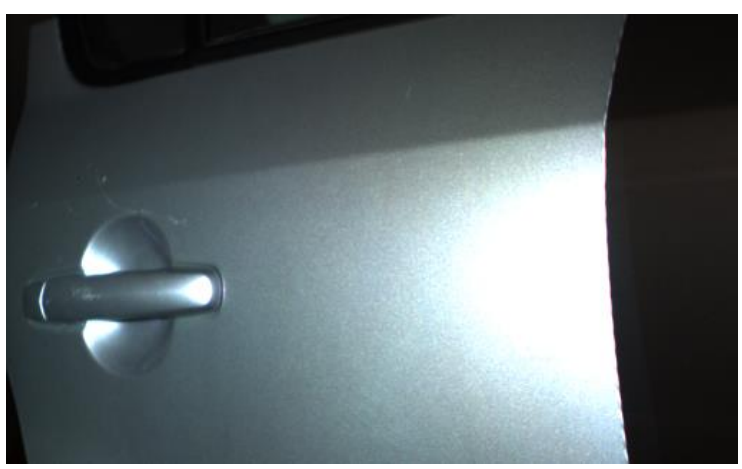

Figure 9

The targeted surface (car door segment)

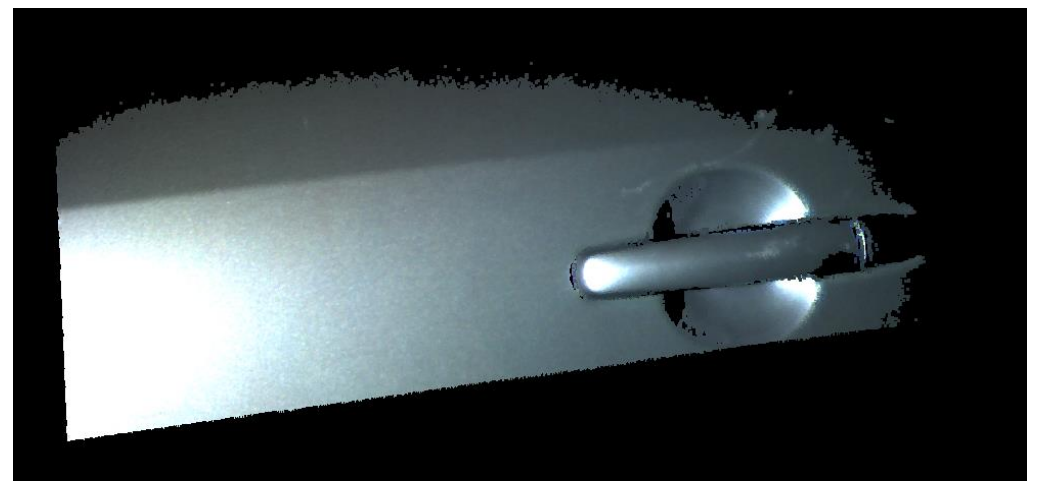

Figure 10

Result of the measured car door segment (View-1) 


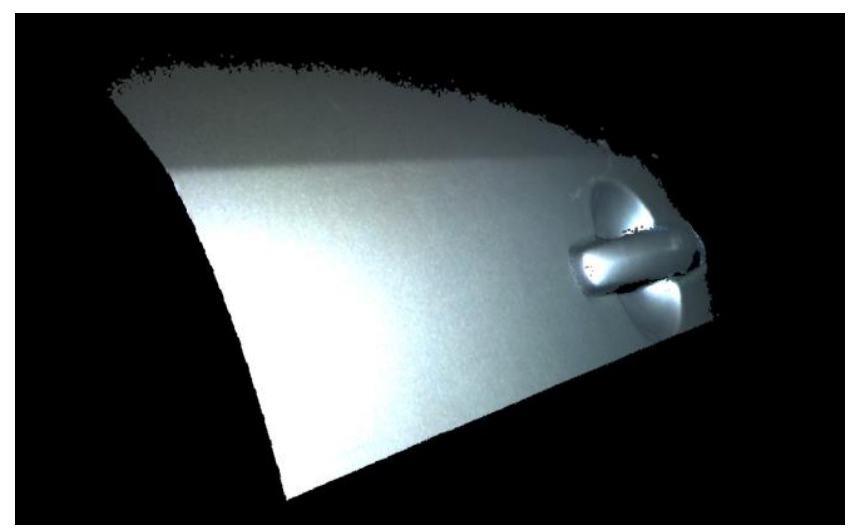

Figure 11

Result of the measured car door segment (View-2)

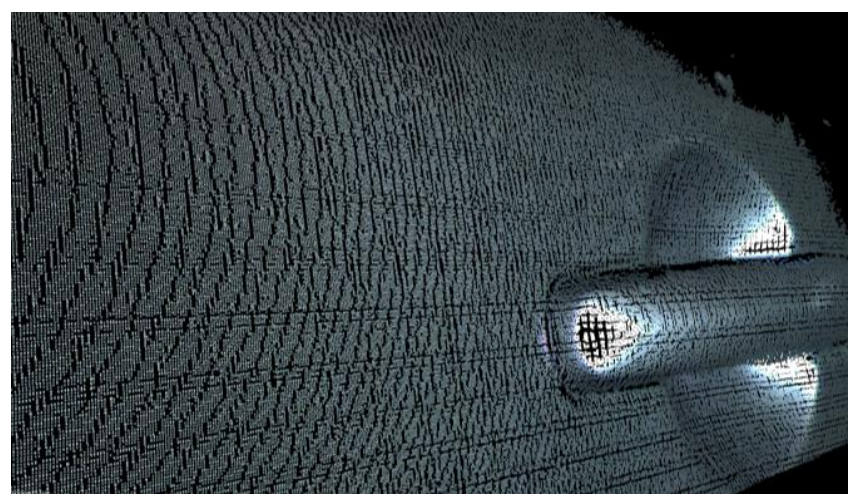

Figure 12

Enlarged point cloud (View-1)

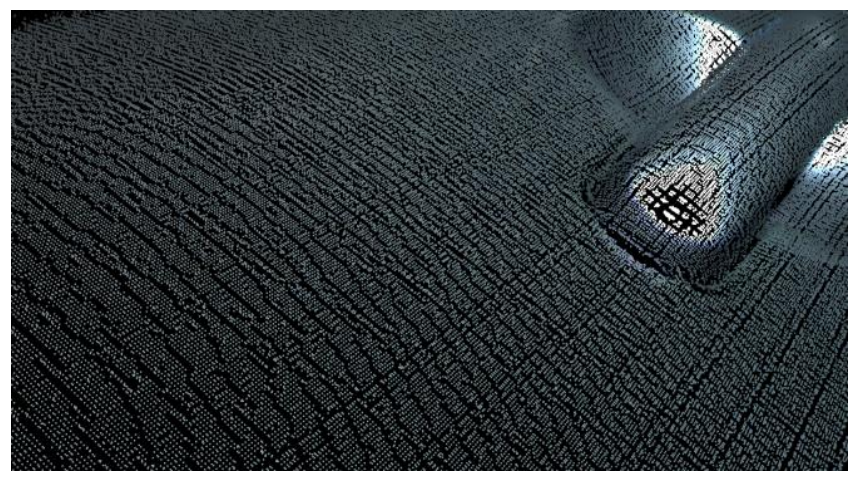

Figure 13

Enlarged point cloud (View-2) 


\section{Conclusions and Future work}

In the paper a tracked camera-projector based 3D measurement system has been proposed. The system is based on so-called "rotating" gray code pattern projection and a multi-camera based tracking system supporting the measurement of complex and large surfaces, as well. As future work the intensity of the projector light is going to be controlled based on the reflectance properties of the target surface. Thermal camera attached to the camera-projector subsystem will be used to perform higher level analysis of the targeted surface.

\section{Acknowledgement}

The project was realized through the support of European Union, with the cofinancing of the European Social Fund TÁMOP-4.2.1.B-11/2/KMR-2011-0001

\section{References}

[1] M. Young, E. Beeson, J. Davis, S. Rusinkiewicz and R. Ramamoorthi, "Viewpoint-Coded Structured Light", IEEE Computer Society Conference on Computer Vision and Pattern Recognition, pp. 1-8, June 2007

[2] E. Lilienblum, B. Michaelis, "Optical 3D Surface Reconstruction by a Multi-Period Phase Shift Method," Journal of Computers, Vol. 2, No. 2, pp. 73-83, 2007

[3] N. Karpinsky, S. Zhang, "High-Resolution, Real-Time 3D Imaging with Fringe Analysis," Journal of Real-Time Image Processing, Springer-Verlag, Vol. 7, Issue 1, pp. 55-66, 2010

[4] Yan Cui, Schuon, S., Chan, D., Thrun, S., Theobalt, C., "3D Shape Scanning with a Time-of-Flight Camera," 2010 IEEE Conference on Computer Vision and Pattern Recognition (CVPR), pp. 1173-1180, 13-18 June 2010

[5] Z. Zhang, "A Flexible New Technique for Camera Calibration," IEEE Transactions on Pattern Analysis and Machine Intelligence, 22(11):13301334,2000

[6] Radu Horaud and Fadi Dornaika, "Hand-Eye Calibration," International Journal of Robotics Research Vol. 14, No. 3, pp. 195-210, 1995

[7] Fang-Hsuan Cheng; Chien-Te Lu; Yea-Shuan Huang, "3D Object Scanning System by Coded Structured Light," Electronic Commerce and Security (ISECS), 2010 Third International Symposium on , Vol., No., pp. 213,217, 29-31 July 2010

[8] Huang Jin; Ma Zi; Hu Ying; Wang Yang, "Robotic 3D Structured Light Scanning System Based on External Axle," Intelligent Computation Technology and Automation (ICICTA), 2008 International Conference on , Vol. 1, No., pp. 1126,1129, 20-22 Oct. 2008 
[9] H B Wu, Y Chen, M Y Wu, C R Guan and X Y Yu. 3D Measurement Technology by Structured Light Using Stripe-Edge-based Gray Code. Institute of Physics Publishing, 2006

[10] M. Gupta, A. Agrawal, A. Veeraraghavan, and S. G. Narasimhan. 2011. Structured light 3D scanning in the presence of global illumination. In Proceedings of the 2011 IEEE Conference on Computer Vision and Pattern Recognition (CVPR '11) IEEE Computer Society, Washington, DC, USA, 713-720

[11] Hani, A. F M; Khoiruddin, A. A.; Walter, N.; Faye, I.; Mun, T. C., "3D Reconstruction Using Spline Inverse Function Analysis," Intelligent and Advanced Systems (ICIAS), $20124^{\text {th }}$ International Conference on , Vol. 1, No., pp. 324,327, 12-14 June 2012

[12] Nikolaus Karpinsky and Song Zhang. 2012. High-Resolution, Real-Time 3D Imaging with Fringe Analysis. J. Real-Time Image Process. 7, 1 (March 2012), 55-66

[13] M. Takeda, "Fourier Fringe Analysis and Its Application to Metrology of Extreme Physical Phenomena: a Review [Invited]," Appl. Opt. 52, 20-29 (2013)

[14] P. Huang and S. Zhang, "Fast Three-Step Phase-Shifting Algorithm," Appl. Applied Optics, Vol. 45, Issue 21, pp. 5086-5091 (2006)

[15] Rovid, A.; Szeidl, L.; Yamage, Y.; Takeshi, M.; Hashimoto, T., "Indoor Real-Time 3D Reconstruction of Crash-tested Car Cabins," Intelligent Systems and Informatics (SISY), $20108^{\text {th }}$ International Symposium on , Vol., No., pp. 257,261, 10-11 Sept. 2010

[16] Li, B.; Holstein, H., "Using k-d Trees for Robust 3D Point Pattern Matching," In Proc. of the Fourth International Conference on 3-D Digital Imaging and Modeling, pp. 95-102, 6-10 Oct. 2003

[18] Zhou, Kun and Hou, Qiming and Wang, Rui and Guo, Baining, ,Real-Time KD-Tree Construction on Graphics Hardware," ACM Trans. Graph., Vol. 27, No. 5, ISSN 0730-0301, pp. 126:1-126:11, 2008

[19] Fernandez, S.; Salvi, J., "Planar-based Camera-Projector Calibration," 2011 $7^{\text {th }}$ International Symposium on Image and Signal Processing and Analysis (ISPA), pp. 633-638, 4-6 Sept. 2011 